\title{
Social Media Usage and Cultural Dimensions: An Empirical Investigation
}

\author{
Majid Dadgar \\ University of San Francisco \\ mdadgar@usfca.edu
}

\author{
Joseph Vithayathil \\ Washington State University \\ joseph.vithayathil@wsu.edu
}

\author{
John Kalu Osiri \\ University of Nebraska-Lincoln \\ jkosiri@unl.edu
}

\begin{abstract}
Cultural attributes of employees affect organizations in several different ways through their impact on organizational goals and decision-making processes. Social media create ample opportunities for organizations to improve competitiveness and efficiency of marketing and communications. We empirically investigate the impact of employee cultural dimensions on social media usage at work and at home. Such a study has not been undertaken before to the best of our knowledge and this would be the first study to connect cultural dimension characteristics of individuals with social media usage. Specifically, we investigate the effect of Power Distance (PD), Uncertainty Avoidance (UA), and Individualism-Collectivism (IC) on the use of popular social media platforms such as Facebook, Twitter, Skype, and LinkedIn. Our results show that certain cultural dimensions predict higher or lower levels of use of specific social media platforms. We provide implications of our results on research and practice.
\end{abstract}

\section{Introduction}

Culture is defined as a combination of common characteristics that affects human responses to the environment [16]. An organizational culture shows professional subculture and the organization's own history [14]. At the collective cultural level people within an organization share and express their thoughts and interests with others [14]. Hofstede [15] argues that culture affects organizations in several different ways through its impact on organizational goals and decisionmaking processes.

People exhibit different levels of distinctive personalities at the individual level within an organizational culture [15]. Technology also plays a major role in the development of organizational structures [14]. Furthermore, organizations conserve and transfer cultural dimensions in variety of ways [33]. Prior research shows that cultural dimensions affect workplace behaviors, attitudes and other organizational outcomes [23]. Orlikowski and Barley [28] argue that information technology and organizational studies can benefit each other by exchanging and analyzing the socio-material properties of their subject matter. The organizational social media literature has focused mainly on disciplinary or domain-based research. Such focus may have hindered possibilities of investigating such important interdisciplinary questions [27].

Social media create a variety of new opportunities for organizations which are not managed well to improve [21]. Organizations can use social media to stay competitive and improve the efficiency of marketing and communications $[1,2,3,10,17,18,25,29]$. Employees use different types of social media platforms in their professional and personal lives, and their use of social media can have an impact on various organizational goals and strategies [20]. In this study we investigate how the cultural dimensions of employees impact their social media usage. Specifically, we investigate the impact of power distance (PD), uncertainty avoidance (UA), and individualism-collectivism (IC) [36]. Such a study has not been undertaken before to the best of our knowledge, and this would be the first study to link social media usage and individual cultural dimensions.

Social media, "a group of Internet-based applications that build on the ideological and technological foundations of Web 2.0 and allow the creation and exchange of User Generated Content" [20:61], is changing the traditional and customary practices in communication, collaboration, consumption, and creation of value at home and at work environments. The use of social media in organizations is growing and this growth is expanding the possibilities for expedient and accessible communication channels [1]. Today, employees in different organizational departments have the capabilities of sharing and creating knowledge using a variety of social media platforms such as Facebook, Twitter, Skype, LinkedIn, and Other Social Media [21]. Social media have been well covered, but the impact of cultural dimensions on social media usage remains, and this area has not been studied. 


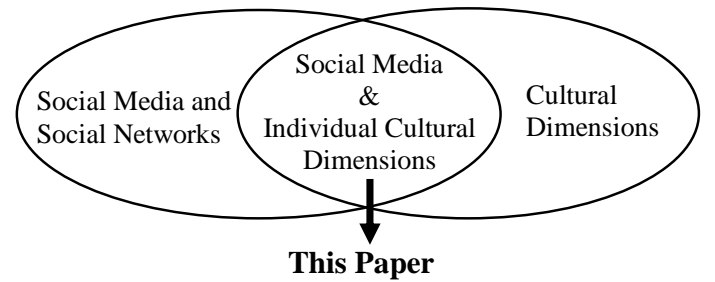

Figure 1. Research Boundaries.

We investigate the effect of cultural dimensions on social media usage in this research study as shown in Figure 1. Specifically, we investigate the effect of PD, UA, and IC on using Facebook, Twitter, Skype, LinkedIn, and other kinds of social media at home and at work.

PD is defined as the extent to which individuals in an organization believe in unequal distribution of power and hierarchies [36].

UA is defined as the extent to which individuals perceive uncertainties in their actions and thoughts and attempt to mitigate their negative impact [16]. Such individuals would request more instruction, rules, and guidance in their work [23].

IC is defined as the extent to which employees in an organization act as individuals than as members of groups [33].

Our findings show that except for using LinkedIn at work, PD predicts use of social media. PD predicts higher use of Facebook, Twitter, Skype, and Other Social Media at work and at home. UA predicts higher use of Skype at work and lower levels of using Skype and LinkedIn at home. IC predicts lower levels of using Skype at work. Other Social Media is a general category in our survey to include all other social media platforms as well as firm-specific or enterprise-specific social media platforms.

Our results suggest that organizations can use social media to target certain marketing campaigns to customers with certain cultural attributes. Organizational culture can be tailored and adapted through systematic and strategic use of social media to connect with employees and for employee use within organizations [37]. Purposeful use of social media in institutions can be enhanced and leveraged by understanding the nuances of understanding employees' cultural dimensions and social media usage. These implications are described in section 6 which is the Discussion section.

The paper is organized as follows. First, we review the theoretical background and relevant literature to develop our hypotheses. Next, we describe our method, data and our model, followed by results and a discussion of the results, their implications, and our conclusion.

\section{Theoretical background, literature review, and hypotheses}

\subsection{Theoretical background}

Information richness is defined as "the ability of information to change understanding within a time interval" [5:560]. Communicative tools, such as social media, that can reduce complexities of communications and create a unified frame of reference in communication transactions are considered rich media [6]. Richness of media is determined by the medium's capability to provide immediate feedback, the variety of information cues and channels, customization to the personal preferences, and the language diversity offered by the medium [5]. Table 1 maps popular social media against media richness criteria.

Prior literature uses Media Richness Theory (MRT) to explain the impact of communicative media on organizational performance $[19,22,26,29,30,32]$. Dennis and Kinney [6] examine the impact of computermediated communication on team decision-making. They define richness of media based on "multiplicity of cues and immediacy of feedback". Koo et al. [24] investigate the manner in which social relationships moderate the impact of media usage on individual performance by drawing on MRT and using empirical analysis. They find that media usage has a positive impact on individual performance, with social factors moderating the degree of this relationship.

Table 1. Social Media against MRT criteria

\begin{tabular}{|l|c|c|c|c|}
\hline MRT & FB & Twitter & Skype & LinkedIn \\
\hline $\begin{array}{l}\text { Immediate } \\
\text { feedback }\end{array}$ & $\mathrm{X}$ & & $\mathrm{X}$ & \\
\hline $\begin{array}{l}\text { Information cues } \\
\text { and channels }\end{array}$ & & & $\mathrm{X}$ & \\
\hline personalization & $\mathrm{X}$ & $\mathrm{X}$ & $\mathrm{X}$ & $\mathrm{X}$ \\
\hline $\begin{array}{l}\text { Language } \\
\text { diversity }\end{array}$ & $\mathrm{X}$ & $\mathrm{X}$ & $\mathrm{X}$ & \\
\hline
\end{tabular}

Among the popular social media platforms, Skype has the highest richness. It provides immediate feedback via video connections, variety of information channels and cue via text, voice, video, and personalization features for text messages, video and voice call settings, and communication channels for text-based and visual languages. LinkedIn is the least rich social medium. It only affords personalizing job profiles and accounts. LinkedIn's communicative features are asymmetric and do not provide immediate feedback and cues. Twitter does not provide immediate feedback, however the "mentions" and "direct messages" can facilitate a 
variety of communication languages. Twitter also has broadcast capability. Twitter enables communication of text-based posts, pictures, and videos among users. Facebook has immediate feedback capabilities via its newsfeed, supports profile personalization, and provides language diversity by its messenger.

Leonardi et al. (2013) examine prior literature to define enterprise social media. They differentiate enterprise social media from traditional media. Their analysis shows that enterprise media function as a platform rather than a channel of communication. Whereas social media have been investigated in the areas of cooperative work and human computerinteraction $[1,4,7,8,9]$, there is a paucity of research in social media and its relationship to individual cultural dimensions.

We analyze the connection between the two theoretical realms of cultural dimensions and social media. Drawing on MRT we examine the impact of cultural dimensions on the use of popular social media platforms such as Facebook, Twitter, Skype, and LinkedIn.

\subsection{Literature review and hypotheses}

Geert Hofstede's works [14,16] from thirty years ago, have inspired several empirical studies of Hofstede's cultural value dimensions. In his view culture is defined as a combination of common characteristics that affect individual human responses to the environment. An organizational culture shows professional subculture and the organization's own history [14]. At the collective cultural level people within an organization share and express their thoughts and interests with others [16]. Hofstede argues that culture affects organizations in several different ways through its impact on organizational goals and decisionmaking processes. He highlights the role of technology as being conducive to the formation of organizational structures. At the individual level within any organizational culture, people display various levels of distinctive personalities [33].

The use and impact of social media in organizations have been investigated in the prior studies. For example, Waters and Lo [35] through a content analysis of Facebook profiles of organizations examine the impact of traditional cultural norms on the communication behavior of organizations in the social media space. Treem and Leonardi [34] investigate the impact of social media usage on organizational communication processes. Segerberg and Bennett [31] examine the impact of Twitter on collective socially and politically motivated actions. The impact of individual cultural dimensions on social media preferences of working individuals has not been investigated in prior research.
Organizations conserve and transfer cultural dimensions in variety of ways [12]. In this study we investigate the effect of cultural dimensions of PD, UA, and IC [36] on the use of popular social media platforms such as Facebook, Twitter, Skype, LinkedIn, and Other Social Media. Other Social Media captures a broad range of enterprise and commercial social media that stand outside popular social media. In the light of innovative advancements in e-commerce and the emergence of companies such Uber and Airbnb we believe these cultural dimensions studied herein are important for organizations.

Power Distance (PD) is defined as the extent to which individuals accept unequal distribution of power and authoritative hierarchies [36]. Individuals having a high PD characteristic are task-oriented and they are less open about their thoughts and feelings with their superiors or managers [23]. However, social media can be a venue where such that individuals with high PD attributes can promote their way of thinking to influence others and simulate the power structure they prefer [11], which they may not enjoy at work or in their personal life. Therefore, we hypothesize:

\section{H1: Higher levels of Power Distance predict higher levels of social media usage.}

Uncertainty Avoidance (UA) is defined as the extent to which individuals perceive uncertainties in their actions and thoughts, and attempt to mitigate their negative impact [16]. Such individuals would request more instruction, rules, and guidance in their work [23]. Individuals who have high UA will use social media to seek more information in order to reduce complexity and uncertainty. Therefore, we hypothesize:

\section{H2: Higher levels of Uncertainty Avoidance predict higher levels of social media usage.}

Individualism-Collectivism (IC) is defined as the extent to which employees in an organization act as individuals than as members of groups [33]. Within an individualism framework people look after themselves and their immediate family members. Within collectivism framework people seek group activities, they trust their group members, enhance their group affiliation, and participate in collective decision making [16]. Individuals with collectivist attributes are more likely to work in teams and with others, and socialize outside work compared to individualists [23]. Such collectivist individuals are more likely to use social media to exchange ideas and share experiences. Therefore, we hypothesize: 
H3: Higher levels of collectivism (IC) predict higher levels of social media usage.

Our research model is illustrated in Figure 2.

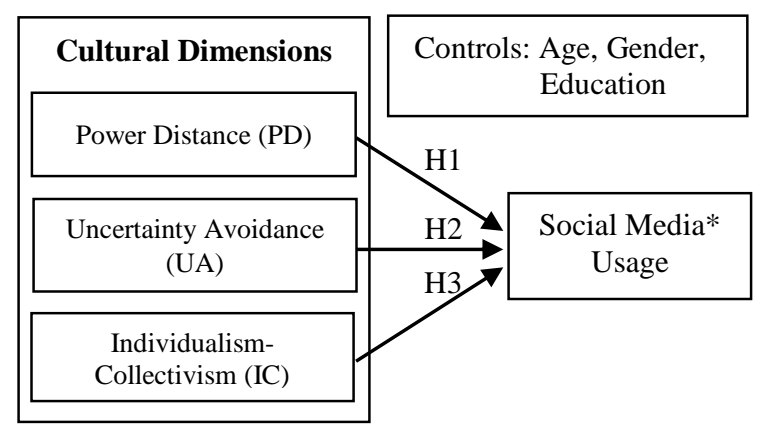

Figure 2. Research model

* Social media tested in our study: Facebook (FB), Twitter, LinkedIn, Skype, and Other Social Media.

\section{Method}

A total of 294 subjects $(\mathrm{N}=294)$ participated in this study. The participants were online students of a public university in the state of Washington who are employed. The majority of students were from Washington, however, some of the participating online students were from other states. Our sample consists of students who are early in their career and familiar with new technologies such as social media. In our data collection we capture Hofstede's cultural dimensions at individual levels in the context of work and use of social media at work and at home. Hence our survey respondents are working students who represent a sample of all social media users with individual cultural attributes. Survey questions are provided in Appendix A. The summary demographics of subjects are provided in Appendix B.

The survey asked 40 questions that covered demographic information, three cultural dimensions, and social media usage at home and at work (see Appendix B). The questions for social media usage measure how frequently subjects use popular social media at work and at home. Our method and findings are limited by the self-reported nature of the survey questions and our subjects, and our regression assumptions.

\subsection{Data and model}

Data were collected by randomly distributing online surveys among participants. Online students who did not have a full time job were eliminated from data analysis. Collected data were aggregated into one dataset. Data screening and cleaning removed the duplicates and incomplete cases. Responses to the social media usage and cultural questions were coded as scale items and responses to demographic questions were coded as categorical using dummy coding. Variables were renamed with user-friendly labels and data dictionary was created for consistent and quick references.

Survey instruments used for measuring cultural dimensions has been developed and validated by Yoo et al. [36] based on Hofstede's cultural dimensions [15] for individuals with cultural dimensions of PD, UA, and IC. The survey questions for social media usage and cultural dimensions are designed based on 5-point Likert scale (see Appendix A).

We use a linear regression model to examine whether cultural dimensions predict the use of social media at work and at home. The model specification is as follows:

$$
\begin{aligned}
\text { SocialMediaUsage }_{i} & =\beta_{0}+\beta_{1 i} \cdot P D_{i}+\beta_{2 i} \cdot U A_{i} \\
& +\beta_{3 i} \cdot I C_{i}+\varepsilon_{i}
\end{aligned}
$$

\section{Results and Discussion}

In the following sections we discuss the results of the regression analysis for each hypothesis. The summary of results is provided in Tables 2 and 3 at the end of this section.

\subsection{H1: higher levels of Power Distance predict higher levels of social media usage.}

Our results show that higher levels of PD predict higher levels of use of Facebook, Twitter, Skype, and Other Social Media at work (see Table 2). Higher levels of PD predict higher levels of using Facebook, Twitter, Skype, LinkedIn, and Other Social Media at home (see Table 3). Therefore, our hypothesis is supported for these relationships. The exception is that higher PD does not predict usage of LinkedIn at work.

Facebook with immediate feedback, personalization, and language diversity [5] provides a rich communicative channel for individuals who rank high in PD dimension. Such individuals use Facebook, for example, to share posts, pictures, and videos to promote their way of thinking. The "Like" feature of Facebook provides them authoritative power to approve or disapprove others' ideas when they use Facebook at home. Individuals with high PD like to make their views known and hence, share or like articles or news from other websites on Facebook for others to see. This power of decision-making provides a simulation of high PD in their personal lives when they use Facebook at work. Therefore, it is attractive to them to replicate such 
a high PD power structure in a virtual social-media world with real people on Facebook.

Approximately 310 million people use Twitter and 100 million of them are active users ${ }^{1}$. MRT shows that Twitter is rich in personalization and languages diversity [5]. Personalizing and exchanging information on a broadcasting platform such as Twitter empowers users with high PD characteristics to diffuse their ideas to a large audience in a short time, and with a precise and concise message. Sending multiple Tweets per day with hashtags will enable high PD individuals to share their ideas quickly and directly with their followers.

Rich media such as Skype provide a full package to impress, influence, and promote one-on-one ideas. Video or conference calls with no charge can be an attractive solution for people characterized as high PD to converse and discuss their thoughts and make normative suggestions on how others should be thinking. Skype is the richest medium that can closely replicate face-to-face communication which is considered the richest means of communication [5].

LinkedIn is a job-specific social medium with the least richness but adequate personalization features. Users can customize their profile and accounts and they can be endorsed by others for their skills and abilities. Individuals with high PD attributes use LinkedIn at work to identify hierarchies and power structures. They use LinkedIn at home to search and find individuals with higher authoritative or managerial powers, or individuals with equal or lower organizational positions. Understanding the power structure at work helps individuals with high PD attributes to better adapt themselves to the work environment.

Other Social Media such as enterprise social media fully satisfy individuals with high PD attributes because they are typically designed to offer limited accessibilities and limited permissions for access to data. Hence, enterprise social media can be highly influenced by the organizational hierarchies and consequently, they are likely to be highly approved and used by individuals endowed with high PD attributes.

\section{H2: Higher levels of Uncertainty Avoidance predict higher levels of social media usage.}

Our results show that higher levels of UA predict higher levels of using Skype at work (see Table 2). Therefore, our hypothesis is supported. However, higher levels of UA predict lower levels of using Skype and LinkedIn at home (see Table 3). Therefore, our hypothesis is not supported for these relationships. UA does not predict use of Facebook, Twitter, LinkedIn, and

\footnotetext{
${ }^{1}$ http://expandedramblings.com/index.php/march-2013-by-thenumbers-a-few-amazing-twitter-stats/
}

Other Social Media at work, and using Facebook, Twitter, and Other Social Media at home.

Skype can translate calls in 7 different languages and users spend more than 3 billion minutes per day on Skype $^{2}$. The paradoxical use of Skype at home and at work by the individuals with high UA characteristics can be a consequence of their inherently uncertain manner of thinking. Such individuals grapple with a duality of using Skype at work and mitigating their uncertainties while they are reluctant to use Skype at home to mitigate uncertainties. Using Skype at home with high richness of information communication informs individuals and could reduce their uncertainties. Paradoxically, the same individual, with high UA, who uses Skype at work for uncertainty reduction does not use Skype to seek more information at home.

Using LinkedIn with the lowest media richness can be effective in mitigating job-related uncertainties but it may not be helpful with other ambiguities that high UA individuals exhibit. It appears that a positive usage of Skype at work and its negative usage at home, spills over to LinkedIn usage in a similar manner and produces lower use of LinkedIn at home. One explanation can be that individuals with high UA prefer specific social media at work for uncertainty reduction. Uncertainty reduction at home or in their personal life may not be a priority compared to uncertainty reduction at work.

\section{H3: Higher levels of Individualism-Collectivism predict higher levels of social media usage.}

Our results show that higher levels of IC (collectivism) predict lower levels of using Skype at work (see Table 2). Therefore, our hypothesis is not supported. IC does not provide any other prediction for the use of other kinds of social media at work. IC does not predict use of social media at home. Therefore, our hypothesis is not supported.

One explanation for this result can be that collectivist individuals prefer face-to-face and in-person communications and socializing with their colleagues and managers at work over using rich media such as Skype at work. Skype might be the richest media for information exchange and communication but it can never replace the conversation and communication in the real word for the collectivist people. Therefore, such individuals may be reluctant to use Skype in the work environment and prefer to take advantage of real faceto-face communications.

\footnotetext{
${ }^{2}$ http://expandedramblings.com/index.php/skype-statistics/
} 
Table 2. Summary of results for social media usage at work and cultural dimensions

\begin{tabular}{|l|l|l|l|l|l|}
\hline DV & IV & $\mathbf{B}$ & $\mathbf{S E}$ & $\mathbf{t}$ & $\mathbf{p}$-value \\
\hline \multirow{4}{*}{ FB_W } & PD & .167 & .098 & 2.870 & $.004 * *$ \\
\cline { 2 - 6 } & UA & -.014 & .102 & -.238 & .812 \\
\cline { 2 - 6 } & IC & .014 & .101 & .244 & .808 \\
\hline \multirow{3}{*}{ Twitter_W } & PD & .266 & .061 & 4.684 & $.000 * * *$ \\
\cline { 2 - 6 } & UA & -.045 & .063 & -.770 & .442 \\
\cline { 2 - 6 } & IC & .062 & .063 & 1.071 & .285 \\
\hline \multirow{3}{*}{ Skype_W } & PD & .062 & .175 & 3.060 & $.002^{* *}$ \\
\cline { 2 - 6 } & UA & .154 & .064 & 2.641 & $.009 * *$ \\
\cline { 2 - 6 } & IC & -.101 & .064 & -1.718 & $.087 *$ \\
\hline \multirow{3}{*}{ OtherSM_W } & PD & .088 & .073 & 1.499 & .135 \\
\cline { 2 - 6 } & UA & .015 & .075 & .243 & .808 \\
\cline { 2 - 6 } & IC & .062 & .075 & 1.030 & .304 \\
\cline { 2 - 6 } & PD & .184 & .083 & 3.183 & $.002 * *$ \\
\cline { 2 - 6 } & UA & .062 & .087 & 1.043 & .298 \\
\cline { 2 - 6 } & IC & .017 & .086 & .293 & .770 \\
\hline \multicolumn{7}{|c|}{$* p<.1 ; * * p<.05 ; * * * p<.001}$. &
\end{tabular}

Table 3. Summary of results for social media usage at home and cultural dimensions

\begin{tabular}{|c|c|c|c|c|c|}
\hline DV & IV & B & SE & $\mathbf{t}$ & p-value \\
\hline \multirow{3}{*}{ FB_H } & PD & .186 & .103 & 3.214 & $.001 * *$ \\
\hline & UA & .036 & .107 & .604 & .546 \\
\hline & IC & -.054 & .105 & -.920 & .358 \\
\hline \multirow{3}{*}{ Twitter_H } & PD & .317 & .089 & 5.680 & $.000 * * *$ \\
\hline & UA & .008 & .092 & .137 & .891 \\
\hline & IC & .040 & .091 & .695 & .488 \\
\hline \multirow{3}{*}{ Skype_H } & PD & .150 & .057 & 2.602 & $.010 * *$ \\
\hline & UA & -.114 & .059 & -1.925 & $.055 *$ \\
\hline & IC & -.021 & .058 & -.351 & .726 \\
\hline \multirow{3}{*}{ LinkedIn_H } & PD & .109 & .080 & 1.891 & $.060 *$ \\
\hline & UA & -.172 & .083 & -2.923 & $.004 * *$ \\
\hline & IC & .053 & .082 & .900 & .369 \\
\hline \multirow{3}{*}{ OtherSM_H } & PD & .164 & .108 & 2.827 & $.005 * *$ \\
\hline & UA & -.033 & .112 & -.550 & .583 \\
\hline & IC & .054 & .110 & .913 & .362 \\
\hline
\end{tabular}

\section{Robustness check}

We perform three robustness checks: i) adding age, gender, and education as controls in the model, ii) testing for the multicollinearity between the predictors, and iii) testing for Heteroscedasticity.

Including age, gender, and education as controls in the regression models do not change any of the associations between the social media usage at home and at work, and cultural dimensions, except for the associations between UA and Skype usage at home, and PD and LinkedIn usage at home, which become nonsignificant. Work uncertainties and hierarchies are not present at home which could affect their associations with certain social media usage at home such as Skype and LinkedIn.
We use collinearity diagnostics in SPSS to test for multicollinearity between the predictors, PD, UA, and IC, for each kind of social media as an outcome variable. Our results show that all the VIF values are below 2 and all the Tolerance values are greater than .95 . Therefore, our results are robust to multicollinearity.

We examine the heteroscedasticity assumptions of our regression models based on the Huber-White heteroscedasticity-consistent estimation of standard errors using the code provided by Hayes and Cai [13]. All the p-values and coefficients in our results are robust to heteroscedasticity except for two relationships that become nonsignificant. The associations between IC and using Skype at work, and PD and using LinkedIn at home become nonsignificant. Therefore, there is a possibility that the significant associations between those variables are inflated by the violation of heteroscedasticity assumptions. This result suggests that IC and Skype, and PD and Skype have weaker relationships, and these two social media have certain characteristics that do not have a normal distribution.

We note that reverse causality is unlikely to be an issue in our model because cultural dimensions and characteristics are formed from an early age and influenced by the environment and the community culture that the individual is exposed to. Hence, it is highly unlikely that cultural dimensions are caused by social media usage.

\section{Discussion}

Our study has several implications for practice. First, organizations can use our findings to target certain users, customers, and clients in certain countries with dominant cultural dimensions of PD, UA, and IC. Specifically, organizations can develop their marketing campaigns in the social media space on the prominent cultural dimensions in their environment. For example, organizations can use Skype-like social media to target certain products or services to customers who exhibit high UA characteristics.

Second, policies regarding the use of popular or enterprise social media can be regulated and controlled for certain individuals in the organizations that hold certain attributes of cultural dimensions. For example, organizations having a dominant collectivist culture can decrease use of social media such as Skype and encourage real-world face-to-face communication and socializing. Organizations that have a high PD dimension can promote the use of social media to enhance and reinforce the organizational power distribution and hierarchies.

Third, our findings can be used to design social media features tailored to certain cultural dimensions. For example, organizations can design features in their 
enterprise social media similar to rich media such as Skype if the UA cultural dimension is dominant in their organization. Customizing social media platforms based on cultural dimensions can benefit individuals working in environments that have those cultural dimensions. Such environments may be countries or organizations. Social media companies could also tailor their products to match specific cultural dimensions.

Our work has a number of limitations. First, our survey is self-reported and the sample population has limitations for generalizability. Therefore, the perceptions of cultural dimensions can vary among contexts and organizations. Second, although we control for certain variables in our regression models, exploring other unobserved variables that can potentially impact social media usage may vary the results, and is beyond the scope of this paper.

\section{Conclusion}

This research is an empirical study to investigate the ability of individual cultural dimensions of PD, UA, and IC to predict the use of popular social media at home and at work. Our findings suggest opportunities for organizations to devise policies that fit employees' cultural dimensions and which promote and reinforce the desired organizational culture. We show that cultural dimensions predict the use of certain social media at work and at home.

Future research could address cross-cultural samples to investigate the use of social media in the counties where certain cultural dimensions are dominant at the individual, organizational, and national levels. Additional cultural dimensions could also be included in future studies to investigate their impact on the use of social media. Social media beyond popular media can be examined to understand how their usage is affected by cultural dimensions. Other measures can be used to test the impact of cultural dimensions such as the differences in the number of posts and Likes on Facebook, or the number and rate of Tweets.

\section{References}

[1] Aral, S., Dellarocas, C., and Godes, D. Social Media and Business Transformation: A Framework for Research. Information Systems Research 24, 1 (2013), 3-13.

[2] Brown, J.J. and Reingen, P.H. Social Ties and Word-of-Mouth Referral Behavior*. Journal of Consumer Research 14, 3 (1987), 350-362.

[3] Clampitt, P.G. and Downs, C.W. Employee Perceptions of the Relationship Between
Communication and Productivity: A Field Study. Journal of Business Communication 30, 1 (1993), 528.

[4] Curtis, L., Edwards, C., Fraser, K.L., et al. Adoption of social media for public relations by nonprofit organizations. Public Relations Review 36, 1 (2010), 90-92.

[5] Daft, R.L. and Lengel, R.H. Organizational Information Requirements, Media Richness And Structural Design. Management Science 32, 5 (1986), 554-571.

[6] Dennis, A.R. and Kinney, S.T. Testing Media Richness Theory in the New Media: The Effects of Cues, Feedback, and Task Equivocality. Information Systems Research 9, 3 (1998), 256-274.

[7] Dewan, S. and Ramaprasad, J. Social media, traditional media, and music sales. MIS Quarterly 38, 1 (2014), 101-121.

[8] Elder, L. and Greene, S. Political information, gender and the vote: the differential impact of organizations, personal discussion, and the media on the electoral decisions of women and men. The Social Science Journal 40, 3 (2003), 385-399.

[9] Ellison, N.B., Gibbs, J.L., and Weber, M.S. The Use of Enterprise Social Network Sites for Knowledge Sharing in Distributed Organizations: The Role of Organizational Affordances. American Behavioral Scientist 59, 1 (2015), 103-123.

[10] Ellison, N.B., Steinfield, C., and Lampe, C. The Benefits of Facebook "Friends:" Social Capital and College Students' Use of Online Social Network Sites. Journal of Computer-Mediated Communication 12, 4 (2007), 1143-1168.

[11] Gibbs, J.L., Eisenberg, J., Rozaidi, N.A., and Gryaznova, A. The "Megapozitiv" Role of Enterprise Social Media in Enabling Cross-Boundary Communication in a Distributed Russian Organization. American Behavioral Scientist 59, 1 (2015), 75-102.

[12] Hanna, R., Rohm, A., and Crittenden, V.L. We're all connected: The power of the social media ecosystem. SPECIAL ISSUE: SOCIAL MEDIA 54, 3 (2011), 265-273.

[13] Hayes, A.F. and Cai, L. Using heteroscedasticityconsistent standard error estimators in OLS regression: 
An introduction and software implementation. 39, (2007), 709-722.

[14] Hofstede, G. Culture and Organizations. International Studies of Management \& Organization 10, 4 (1980), 15-41.

[15] Hofstede, G. Culture's consequences: Comparing values, behaviors, institutions, and organizations across nations. Sage, Thousand Oaks, CA, 2001.

[16] Hofstede, G., Neuijen, B., Ohayv, D.D., and Sanders, G. Measuring Organizational Cultures: A Qualitative and Quantitative Study across Twenty Cases. Administrative Science Quarterly 35, 2 (1990), 286-316.

[17] Jansen, B.J., Zhang, M., Sobel, K., and Chowdury, A. Twitter power: Tweets as electronic word of mouth. Journal of the American Society for Information Science \& Technology 60, 11 (2009), 2169-2188.

[18] Jones, P., Comfort, D., and Hillier, D. What's in store? Retail marketing and corporate social responsibility. Marketing Intelligence \& Planning 25, 1 (2007), 17-30.

[19] Kahai, S.S. and Cooper, R.B. Exploring the Core Concepts of Media Richness Theory: The Impact of Cue Multiplicity and Feedback Immediacy on Decision Quality. Journal of Management Information Systems 20, 1 (2003), 263-299.

[20] Kaplan, A.M. and Haenlein, M. Users of the world, unite! The challenges and opportunities of Social Media. Business Horizons 53, (2010), 59-68.

[21] Kietzmann, J.H., Hermkens, K., McCarthy, I.P., and Silvestre, B.S. Social media? Get serious! Understanding the functional building blocks of social media. 54, 3 (2011), 241-251.

[22] Kim, S. and Lee, H. The Impact of Organizational Context and Information Technology on Employee Knowledge-Sharing Capabilities. Public Administration Review 66, 3 (2006), 370-385.

[23] Kirkman, B.L., Lowe, K.B., and Gibson, C.B. A Quarter Century of "Culture's Consequences": A Review of Empirical Research Incorporating Hofstede's Cultural Values Framework. Journal of International Business Studies 37, 3 (2006), 285-320.

[24] Koo, C., Wati, Y., and Jung, J.J. Examination of how social aspects moderate the relationship between task characteristics and usage of social communication technologies (SCTs) in organizations. International Journal of Information Management 31, 5 (2011), 445-459.

[25] Lee, K., Oh, W.-Y., and Kim, N. Social Media for Socially Responsible Firms: Analysis of Fortune 500's Twitter Profiles and their CSR/CSIR Ratings. Journal of Business Ethics 118, 4 (2013), 791-806.

[26] Luo, X. and Zhang, J. How Do Consumer Buzz and Traffic in Social Media Marketing Predict the Value of the Firm? Journal of Management Information Systems 30, 2 (2013), 213-238.

[27] Murthy, D. Introduction to the Special Issue on Social Media, Collaboration, and Organizations. American Behavioral Scientist 59, 1 (2015), 3-9.

[28] Orlikowski, W.J. and Barley, S.R. Technology And Institutions: What Can Research On Information Technology And Research On Organizations Learn From Each Other? MIS Quarterly 25, 2 (2001), 145165.

[29] Rapp, A., Beitelspacher, L., Grewal, D., and Hughes, D. Understanding social media effects across seller, retailer, and consumer interactions. Journal of the Academy of Marketing Science 41, 5 (2013), 547566.

[30] Schniederjans, D., Cao, E.S., and Schniederjans, M. Enhancing financial performance with social media: An impression management perspective. 1 . Social Media Research and Applications 2. Theory and Applications of Social Networks 55, 4 (2013), 911918.

[31] Segerberg, A. and Bennett, W.L. Social Media and the Organization of Collective Action: Using Twitter to Explore the Ecologies of Two Climate Change Protests. The Communication Review 14, 3 (2011), 197-215.

[32] Smits, M. and Mogos, S. The Impact Of Social Media On Business Performance. ECIS, (2013).

[33] Taras, V., Kirkman, B.L., and Steel, P. Examining the impact of Culture's consequences: A three-decade, multilevel, meta-analytic review of Hofstede's cultural value dimensions. Journal of Applied Psychology 95, 3 (2010), 405-439.

[34] Treem, J.W. and Leonardi, P.M. Social Media Use in Organizations Exploring the Affordances of 
Visibility, Editability, Persistence, and Association. Annals of the International Communication Association 36, 1 (2013), 143-189.

[35] Waters, R.D. and Lo, K.D. Exploring the Impact of Culture in the Social Media Sphere: A Content Analysis of Nonprofit Organizations' Use of Facebook. Journal of Intercultural Communication Research 41, 3 (2012), 297-319.

[36] Yoo, B., Donthu, N., and Lenartowicz, T. Measuring Hofstede's Five Dimensions of Cultural Appendix A - Survey Questions

Q1. Are you currently working?

1. Yes

2. No

Demographics (there are 15 demographic questions. Full demographic questions can be provided upon request)

Q1. What is your age group (years)?

1. less than 20

2. 20 to less than 35

3. 35 to less than 50

4. 50 to less than 65

5. 65 and higher

Q2. What is your gender

1. Female

2. Male

Q3. What is your highest educational degree?

1. High School

2. Community College

3. Four Year College

4. Graduate School

Social Media Usage (there are 10 questions on social media. Full social media questions can be provided upon request)

Q1. Rate your weekly use of Facebook at Home

1. Very Low or None

2. Low

3. Moderate

4. High

5. Very High or Always

Q2. Rate your weekly use of Twitter at Work

1. Very Low or None

2. Low

3. Moderate

4. High

5. Very High or Always
Values at the Individual Level: Development and Validation of CVSCALE. Journal of International Consumer Marketing 23, 3/4 (2011), 193-210.

[37] Yu, Y., Duan, W., and Cao, Q. The impact of social and conventional media on firm equity value: A sentiment analysis approach. 1. Social Media Research and Applications 2. Theory and Applications of Social Networks 55, 4 (2013), 919-926.

\section{Cultural Dimensions}

Power Distance

Q1. Indicate your agreement with the statement: People in higher positions should make most decisions without consulting people in lower positions

1. Strongly Disagree

2. Disagree

3. Moderately Agree

4. Agree

5. Strongly Agree

Q2. Indicate your agreement with the statement: People in higher positions should not ask the opinions of people in lower positions too frequently

1. Strongly Disagree

2. Disagree

3. Moderately Agree

4. Agree

Strongly Agree

Q3. Indicate your agreement with the statement: People in higher positions should avoid social interaction with people in lower positions

1. Strongly Disagree

2. Disagree

3. Moderately Agree

4. Agree

5. Strongly Agree

Q4. Indicate your agreement with the statement: People in lower positions should not disagree with decisions made by people in higher positions

1. Strongly Disagree

2. Disagree

3. Moderately Agree

4. Agree

5. Strongly Agree

Q5. Indicate your agreement with the statement: People in higher positions should not delegate important tasks to people in lower positions

1. Strongly Disagree

2. Disagree

3. Moderately Agree

4. Agree 
5. Strongly Agree

Uncertainty Avoidance

Q6. Indicate your agreement with the statement: It is important to have instructions spelled out in detail so that I always know what I'm expected to do

1. Strongly Disagree

2. Disagree

3. Moderately Agree

4. Agree

5. Strongly Agree

Q7. Indicate your agreement with the statement:

Rules and regulations are important because they inform me of what is expected of me

1. Strongly Disagree

2. Disagree

3. Moderately Agree

4. Agree

5. Strongly Agree

Q8. Indicate your agreement with the statement:

Standardized work procedures are helpful

1. Strongly Disagree

2. Disagree

3. Moderately Agree

4. Agree

5. Strongly Agree

Q9. Indicate your agreement with the statement: Instructions for operations are important

1. Strongly Disagree

2. Disagree

3. Moderately Agree

4. Agree

5. Strongly Agree

Individualism (1) to Collectivism (5)

Q10. Indicate your agreement with the statement:

Individuals should sacrifice self-interest for the group

1. Strongly Disagree

2. Disagree

3. Moderately Agree

4. Agree

5. Strongly Agree

Appendix B. Demographics of the subjects

\begin{tabular}{|l|l|l|}
\hline Variable & $20-35$ years old & Percentage \\
\cline { 2 - 3 } Age & $35-50$ years old & 72 \\
\cline { 2 - 3 } & $50-65$ years old & 25 \\
\hline \multirow{4}{*}{ Gender } & Female & 60 \\
\cline { 2 - 3 } & Male & 40 \\
\hline \multirow{4}{*}{ Education } & High school & 10 \\
\cline { 2 - 3 } & Community college & 45 \\
\cline { 2 - 3 } & Four year college & 44 \\
\cline { 2 - 3 } & Graduate degree & 1 \\
\hline
\end{tabular}

Q11. Indicate your agreement with the statement: Individuals should stick with the group even through difficulties

1. Strongly Disagree

2. Disagree

3. Moderately Agree

4. Agree

5. Strongly Agree

Q12. Indicate your agreement with the statement: Group welfare is more important than individual rewards

1. Strongly Disagree

2. Disagree

3. Moderately Agree

4. Agree

5. Strongly Agree

Q13. Indicate your agreement with the statement: Group success is more important than individual success

1. Strongly Disagree

2. Disagree

3. Moderately Agree

4. Agree

5. Strongly Agree

Q14. Indicate your agreement with the statement: Individuals should only pursue their goals after considering the welfare of the group

1. Strongly Disagree

2. Disagree

3. Moderately Agree

4. Agree

5. Strongly Agree

Q15. Indicate your agreement with the statement: Group loyalty should be encouraged even if individual goals suffer

1. Strongly Disagree

2. Disagree

3. Moderately Agree

4. Agree

5. Strongly Agree 